\title{
EEG-Based Epilepsy Detection and Prediction
}

\author{
Kavya Devarajan, E. Jyostna, K. Jayasri, and Vinitha Balasampath
}

\begin{abstract}
Epilepsy is a fatal condition which is caused as a result of disorder in the nervous system. Approximately $30 \%$ of individuals with epilepsy have refractory seizures that cannot be controlled by current pharmacological treatment measures. For such patients, responsive Neuro-stimulation prior to a seizure may lead to greater efficacy when compared to current treatments. This paper presents a fractal-dimension based algorithm implemented in MATLAB software that alerts epileptic patients for treatment and take preventive measures before the onset of seizure. It also gives an account of the current state of this research field, point towards possible future developments and propose methodological guidelines for future studies on seizure prediction.
\end{abstract}

Index Terms-Brain, electroencephalogram, entropy, epilepsy, epileptic seizure, seizure, signal processing, therapeutic, prediction.

\section{INTRODUCTION}

Epilepsy is a neurological disorder characterized by recurrent seizures as a result of abnormal electrical discharges in the brain. The condition affects approximately $1-2 \%$ of the world's population. Two-thirds of the patients achieve sufficient seizure control from anticonvulsive medication, and another $8-10 \%$ could benefit from surgery. For the remaining $30 \%$ of patients, no sufficient treatment is currently available [1].

Prediction of epilepsy is essential, especially for ICU patients, who persistently experience convulsions due to the overdose of drugs, patients who develop non-convulsive seizures and those who cannot be treated by drugs.

The sudden and conceivably unpredictable nature of seizures is one of the most disabling aspects of Epilepsy. A method that can predict the occurrence of seizures from the electroencephalogram (EEG) of epilepsy patients would open new therapeutic possibilities[2]. Treatment concepts could move from preventive strategies (e.g. Long-term medication with anti-epileptic drugs) towards an EEG-triggered on-demand therapy or other stimulation in an attempt to reset brain dynamics to a state that will no longer develop into a seizure.

There is a specific pattern called the burst suppression waveform that can clearly distinguish itself from the non-seizure time-periods.Fig 1.1 shows the initiation of the symptoms of epilepsy. The onset is characterized by increased frequency and high amplitude values when compared to non-epileptic waveform.(Fig.1.2). Fig. 1.3 represents the post-ictal phase where the burst-supression waveform resides to the normal EEG pattern.Hence, by studying these unique features of the inter-ictal waveform

Manuscript received April 10, 2013, revieed June 10, 2013.

Kavya Devarajan is with the College of Engineering, Gundy, Anna University, Chennai, India (e-mail: kavyadevarajan@gmail.com). and pre-ictal changes, prediction of the onset of epilepsy can be attained. Suitable parameters that relate to the pattern have to be extracted and processed before observing the trend.

Literature review relating to the study has analyzed the four major analysis methods, namely; time domain, frequency domain, time-frequency domain and non-linear analysis. Results show that analysis methodologies involving wavelet decomposition, a time-frequency domain approach, have more accuracy, resolution and sensitivity. Frequency domain analysis fails to whereas time domain lacks efficient analysis of. The EEG data is therefore preprocessed and analyzed using wavelet techniques for extraction of parameters. The parameters define the pre-ictal period of epilepsy, where symptoms called as auras are characterized and guide us to predict the onset of seizure.

The successful development of light weight, novel cost-effective wearable life-saver prediction device will help millions of victims of epilepsy around the globe. With the device in possession, an epilepsy victim can move around freely like normal people sans worries.
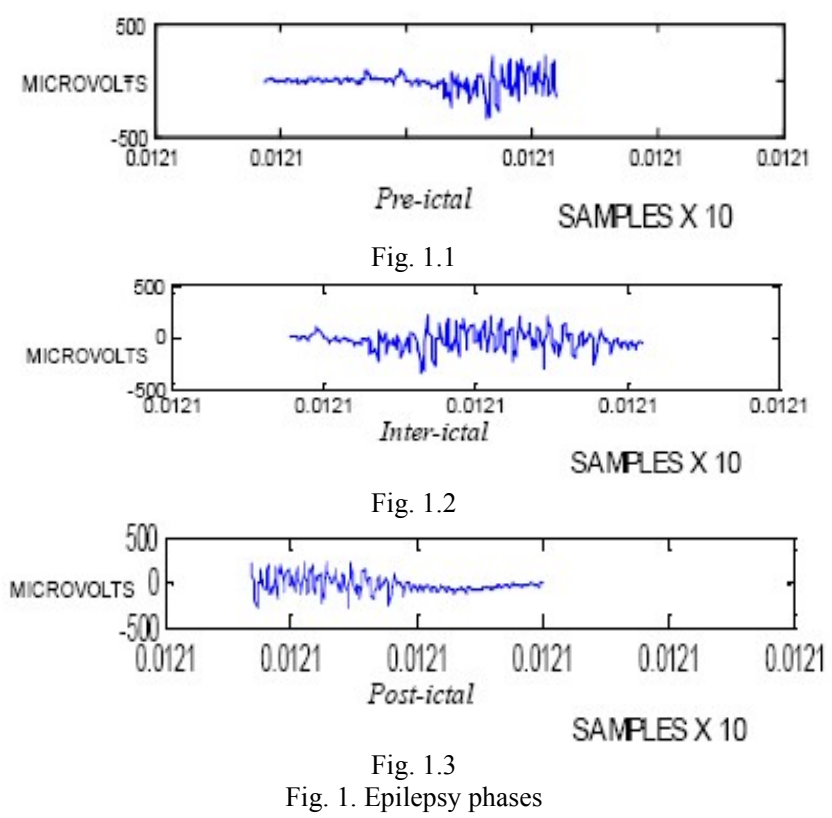

\section{MATERIALS AND METHODS}

\section{A. Data Resources}

In this study, database collected at the Children's Hospital Boston, consisting of EEG recordings of pediatric subjects with intractable seizures was investigated. Subjects were monitored for up to several days following withdrawal of anti-seizure medication in order to characterize their seizures and assess their candidacy for surgical intervention. Recordings were collected from 10 subjects ( 5 males, ages 3-22; and 5 females, ages 1.5-19). All signals were sampled 
at 256 samples per second with 16-bit resolution. Data was available for 22 channels, each file with a length of one hour [3].

SEIZURE DETECTION

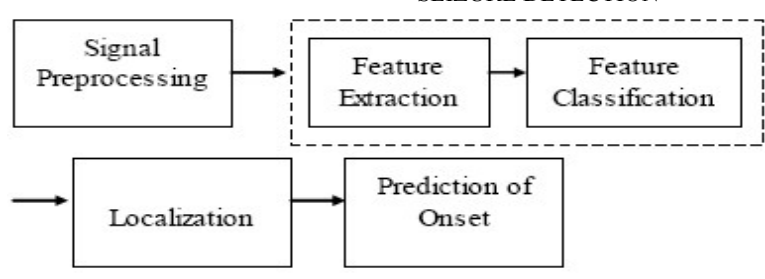

Fig. 2. General block diagram

The algorithm for epileptic prediction requires certain preprocessing steps and analysis methods to be performed, before its development. They can be enlisted as follows: (1) Signal Preprocessing (2) Feature Extraction (3) Feature Classification (4) Localization in brain sites (5) Prediction of onset. The block diagram given in Fig. 2 represents these steps.

Signal preprocessing involves the basic steps of filtering of any biological signals in order to remove noise and artifacts associated with the irrelevant physiological activity. The data available in Physionet database has already been preprocessed and is noise-free.

\section{B. Block Diagram}

Detection of seizures is an important step in the development of the seizure prediction algorithm. The efficiency of the software to differentiate epileptic features from normal EEG with high accuracy and least false rate influence the ability to correctly predict the onset of seizure. Thus, it is crucial to test the system if it displays the results with high sensitivity and specificity, else the prediction and control of seizure may cause unnecessary harm to the patient. It involves the sub-parts feature extraction, classification and thresholding, which will be covered upon in detail.

The algorithm should also be capable to localize the regions from which the seizures originate. In order to prevent epilepsy from occurring, brain pacemakers have to control the brain sites at which the onset takes place. Provoking other brain sites might cause other undesirable effects. Thus, a successful algorithm must not only predict correct seizure arrival but also be able to locate the seizure points to be controlled.

\section{Seizure Detection}

Among the methodologies for seizure detection, Time-frequency domain analysis (wavelet decomposition) has shown to have more accurate results with increased sensitivity and specificity. This is because traditional methods of Fourier analysis have a Considerable trade-off between time and frequency resolutions. Time domain analysis has low sensitivity in high frequency range.. Whereas, time frequency domain analysis such as wavelet analysis is best suited for non-stationary signals. Considering these advantages, EEG data was decomposed using Daubechies wavelet-5 into different frequency ranges (Fig.3). Wavelet decomposition allows optimal selection of decomposition levels with minimum entropy values, high power spectral density, pertaining to epileptic waveform. The desired frequency range thus obtained is from 0 to $16 \mathrm{Hertz}$ in-line with research that has shown that region with low frequency waves are more prone to abnormal brain activities[4].

For the detection of epilepsy, numerous non-parametric features such as energy of wavelet coefficients and instantaneous power were computed to differentiate between normal, background (pre-ictal) and epileptic EEG signals. The choice of these features was based upon physiologic changes in the EEG usually associated with epilepsy such as spikes in subclinical seizures and increased power and energy in the signal [4]. The selected features were also chosen to be computationally efficient and have potential for on-line implementation in low-power, implantable environments[5].

Thus, accumulated signal energy, instantaneous power, the rate of subclinical seizure-like bursts and bursts of long-term energy all increased as seizures approached. Then, the signals are employed suitable thresholds to detect the seizure interval. This is represented in Fig.4. The output of the signal analysis after wavelet decomposition and thresholding for various features are given in Fig.5 and Fig.6. Table I shows the difference in energy percentages in seizure and non-seizure signals so as to distinguish them.
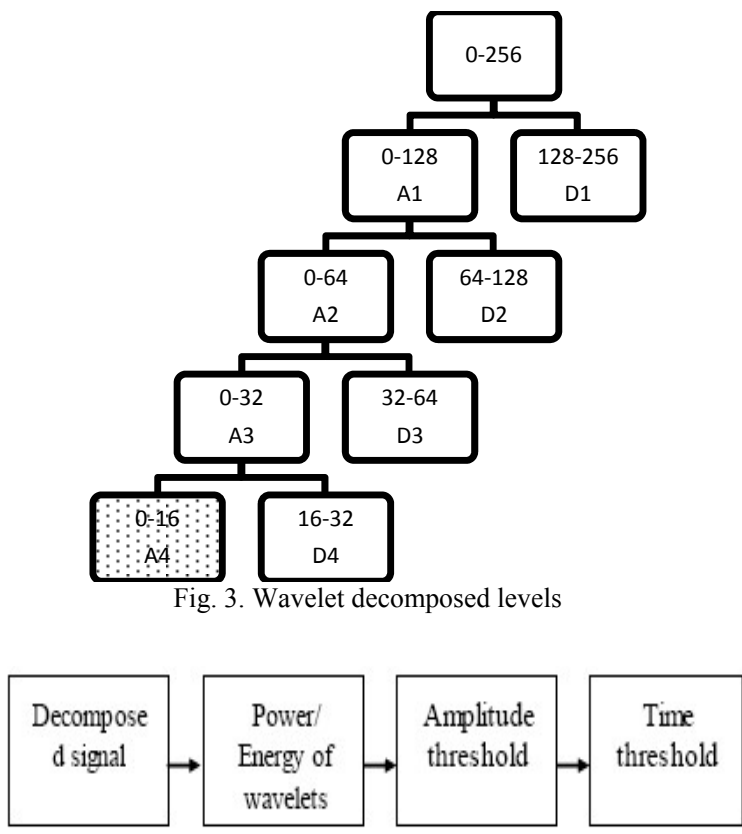

Fig. 4. Detection block diagram
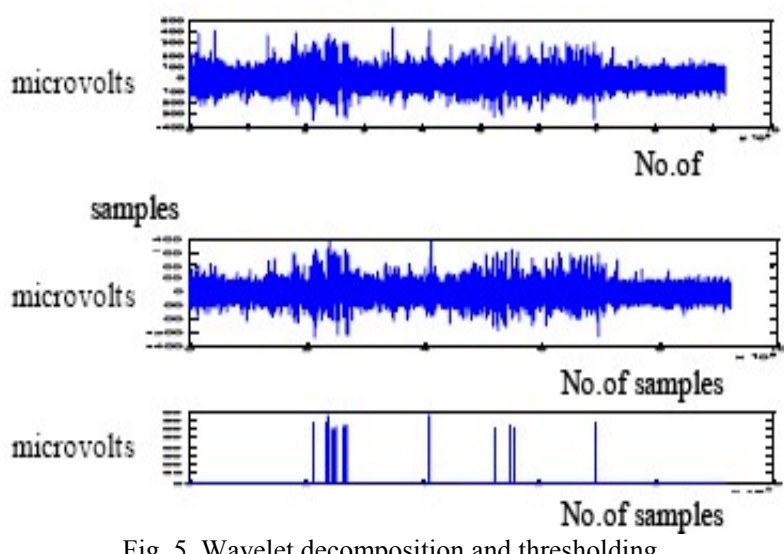

Fig. 5. Wavelet decomposition and thresholding 


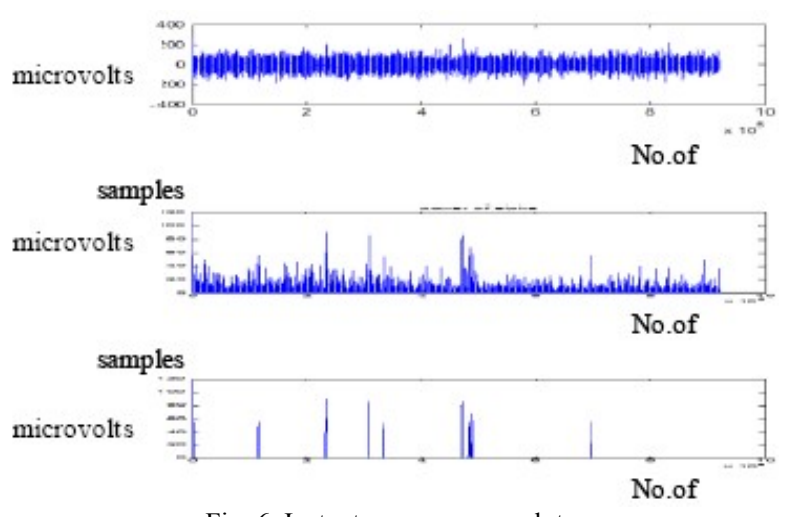

Fig. 6. Instantaneous power plot

TABLE I: ENERgy IN PERCENTAGE

\begin{tabular}{|l|l|l|}
\hline PATIENT & Nonseizure (Energy) & Seizure (Energy) \\
\hline PATIENT 1 & $2.3 \%$ & $7.0 \%$ \\
\hline PATIENT 2 & $2.1 \%$ & $7.4 \%$ \\
\hline PATIENT 3 & $2.3 \%$ & $6.0 \%$ \\
\hline PATIENT 4 & $2.5 \%$ & $8.0 \%$ \\
\hline PATIENT 5 & $2.0 \%$ & $6.7 \%$ \\
\hline PATIENT 6 & $2.2 \%$ & $8.0 \%$ \\
\hline PATIENT 7 & $2.08 \%$ & $6.8 \%$ \\
\hline PATIENT 8 & $2.85 \%$ & $7.3 \%$ \\
\hline PATIENT 9 & $2.6 \%$ & $7.6 \%$ \\
\hline PATIENT 10 & $2.4 \%$ & $7.3 \%$ \\
\hline
\end{tabular}

\section{Localization of Seizures}

Precise focus localization and automatic online seizure detection from intracranial EEG recordings are critical for closed-loop intervention, but remain challenging problems. For as many as $30 \%$ of epilepsy patients, Seizures are poorly controlled with medication alone. For some of these patients where brain region responsible for seizure onset may be removed surgically, it requires accurate delineation of the seizure onset region[6]. This phenomenon may in the long term allow determining the seizure onset areas from non-seizure EEG, which in turn would enable shorter hospitalizations or even avoidance of semi-chronic implantations all-together.

A successful outcome of epilepsy surgery depends on the localization of the epileptic tissue which is structurally and functionally abnormal. The availability of reliable methods of seizure prediction and localization could enhance the quality and safety of patients with epilepsy; facilitate implementation of short-term interventions to abort a seizure.

\section{E. Prediction of Epileptic Seizures}

A number of quantitative features derived from the EEG, each with different the oretical bases, are useful for interpreting complex pathophysiological phenomena [2]. Hurst exponent, is one such can help interpret epileptic and their neurodynamics. The Hurst Exponent is a numerical estimate of the predictability of a time series. It is defined as the relative tendency of a time series to either regress to a long term mean value or 'cluster' in a direction [7]. It has been shown to be useful to characterize an epileptic brain shows that the epileptic brain is long term anticorrelated whereas, the normal brain is more or less stochastic.

The aim of this study was to find characteristic changes in the Hurst exponent values. Hurst exponent $(H)$ is used to describe a fractal self-affine object, because it indicates the level of roughness of curves[8]. $\mathrm{H}$ is used to determine if an EEG series has a fractal behavior and it measures the intensity of dependence.

It is said that an analyzed phenomena is random (Brownian motion) when $H=0.5$; it is persistent when $0.5<H<1$ (invariance scale is associated to positive correlations in long range, it means an increasing period is followed with a similar one), and finally it is anti-persistent when $0<H<0.5$ (invariance in the scale associated to negative correlations in long range, it means an increasing period is followed by a decreasing one)[8]. It was found that the $\mathrm{H}$ values of EEG seizure activity significantly decreased before initiation of ictal activity, and the $\mathrm{H}$ value increased after ictal attacks.

\section{$F$. Calculation of the Hurst Exponent}

The scaling properties of a given discrete time series $X=$ $\{X 1, X 2, . . X N\}$ can be expressed through the dependence of the autocorrelation function $C(k)$ vs the Hurst exponent $(H)$ in terms of a large-time lag. [8]

1) Calculate the mean

$$
M=1 / n \sum X i
$$

2) Create a mean-adjusted series

$$
Y t=X t=m \text { for } t=1,2, \ldots, n
$$

3) Calculate the cumulative deviate series

$$
Z t=\sum Y i \text { for } \mathrm{t}=1,2, \ldots, n
$$

4) Compute the range

$$
R(n)=\max (Z 1, Z 2, \ldots, Z n)-\min (Z 1, Z 2, \ldots, Z n)
$$

5) Compute the standard deviation

$$
S_{t}=\sqrt{\frac{1}{t} \sum_{i=1}^{t}\left(X_{i}-u\right)^{2}} \quad t=1,2, \ldots, n
$$

6) Calculate the rescaled range and average over all the partial time series of length $n$.

$$
\left(\frac{R}{S}\right)_{t}={\frac{R_{t}}{S_{t}}}_{t=1,2, \ldots \ldots, n}
$$

The Hurst exponent is estimated by fitting the power law to the data. This can be done by plotting the logarithm of (1) as a function of $\log n$. [7]. The asymptotic value of $H$ was determined for a 1-Sec-long period of observation with sampling Rate of $256 \mathrm{~Hz}$. It was found that, starting from the pre-ictal duration and thereafter, the $H$ value started to decline continuously, with no global variation until the beginning of an epileptiform attack, when it reached a minimum value (Fig. 7.1 and Fig. 7.2). This period before the beginning of the epileptiform attack is called the critical period. This critical period is the time before which we can predict the onset of the seizure. 

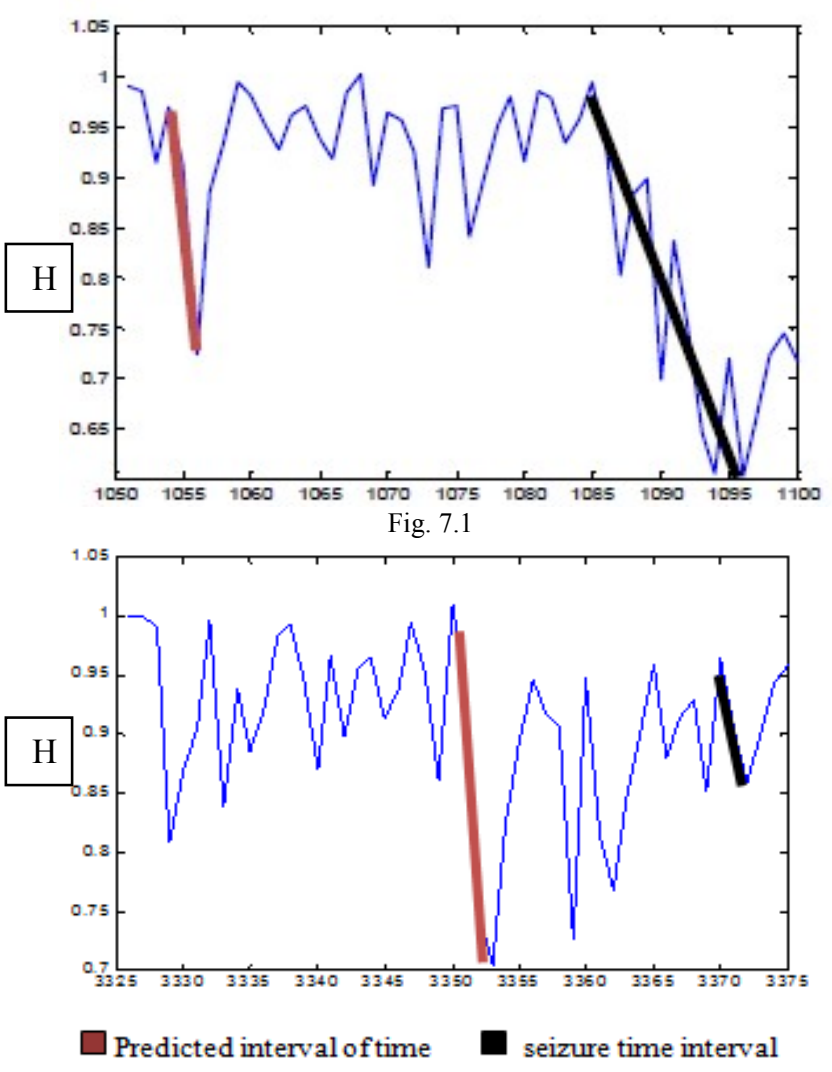

Fig. 7.2

Fig. 7. Slope changes of Hurst exponent values

\section{RESULTS AND DISCUSSIONS}

The performance of the seizure onset predictor was evaluated in 20 subjects (5 males and 15 females). After preprocessing, the data were first tested for its accuracy in detection of seizure. Wavelet decomposition was then employed for classification and features such as energy, power and entropy to characterize the epileptiform.

Additional abnormalities were removed using time thresholding. The detection algorithm presented true results for over 17 patients attributing to an accuracy of $87.5 \%$. The algorithm also proved to be efficient to detect even small durations of additional epileptic seizure waveforms.Hurst exponent $(H)$, a chaotic measure, is then used to characterize the complexity of the signal. The Hurst values were plotted, in which we observed a unique trend in the EEG activity with respect to Epilepsy duration. We have found that the Hurst exponent analysis not only distinguishes epileptic EEG from normal EEG, but also plots significant changes before and after the seizure. More precisely, it was found that the $H$ values of EEG seizure activity significantly decreased before initiation of ictal activity, and the $\mathrm{H}$ value increased after ictal attacks. At non-seizure interval times, the Hurst exponent values limited to a small range of values. This unique pattern present few seconds prior to onset of seizure has been exploited for the prediction of Epilepsy. The trend has been observed in all localized channels involving seizures at a pre-ictal duration varying from two to sixty seconds. The results have been presented for twenty patients in Table II. Following the Hurst exponent analysis and consistent results it is proved that epileptic brain activity shows long range anticorrelation or antipersistency. In other words, the behavior of the EEG time series has been found to be a "persistent" behavior, which means that an increasing or a decreasing trend of the time series is characteristically present around the seizure interval.

This feature may be attributed to the fact that the signal possesses much greater predictability when long-range correlation effects predominate. Thus, a trend toward increasing or decreasing the amplitude of the signal in the past will be followed by the same trend in the future, as is in the case where the epileptic attack develops. In particular, when $\mathrm{H} \rightarrow 1$, these effects bear the name long-memory effects, because, roughly speaking, the signal "remembers" its previous state. [9]

Therefore, Hurst Exponent provides a method of classifying time series, which can be useful in identifying values of short term predictability.

TABLE II: PRE-SEIZURE DURATION

\begin{tabular}{|l|l|l|l|}
\hline PATIENT & \multicolumn{1}{|l}{ Detected Time } & Predicted Time & Hurst $(\mathrm{h})$ \\
\hline Patient1 & $1015-1066 \mathrm{~s}$ & $1007-1008 \mathrm{~s}$ & $0.9-0.7$ \\
\hline Patient2 & $3369-3378 \mathrm{~s}$ & $3349-3350 \mathrm{~s}$ & $0.9-0.7$ \\
\hline Patient3 & $1665-1726 \mathrm{~s}$ & $1661-1664 \mathrm{~s}$ & $0.9-0.7$ \\
\hline Patient4 & $1039-1061 \mathrm{~s}$ & $1044-1045 \mathrm{~s}$ & $0.8-0.66$ \\
\hline Patient5 & $3025-3140 \mathrm{~s}$ & $3000-3002 \mathrm{~s}$ & $0.9-0.66$ \\
\hline Patient 6 & $362-414 \mathrm{~s}$ & $357-358 \mathrm{~s}$ & $0.9-0.7$ \\
\hline Patient 7 & $1086-1196 \mathrm{~s}$ & $1053-1054 \mathrm{~s}$ & $0.9-0.72$ \\
\hline Patient 8 & $3285-3381 \mathrm{~s}$ & $3264-3265 \mathrm{~s}$ & $0.8-0.77$ \\
\hline Patient 9 & $2856-3046 \mathrm{~s}$ & $2820-2821 \mathrm{~s}$ & $0.9-0.83$ \\
\hline Patient 10 & $627-635 \mathrm{~s}$ & $592-594 \mathrm{~s}$ & $0.9-0.71$ \\
\hline Patient 11 & $2077-2121 \mathrm{~s}$ & $2031-2032 \mathrm{~s}$ & $0.9-0.64$ \\
\hline Patient 12 & $299-377 \mathrm{~s}$ & $273-274 \mathrm{~s}$ & $0.9-0.75$ \\
\hline Patient 13 & $94-123 \mathrm{~s}$ & $60-62 \mathrm{~s}$ & $0.9-0.82$ \\
\hline Patient 14 & $1288-1344 \mathrm{~s}$ & $1242-1244 \mathrm{~s}$ & $0.9-0.6$ \\
\hline Patient 15 & $3489-3527 \mathrm{~s}$ & $3477-3479 \mathrm{~s}$ & $0.9-0.7$ \\
\hline Patient 16 & $5299-5361 \mathrm{~s}$ & $5286-5287 \mathrm{~s}$ & $0.95-0.8$ \\
\hline Patient 17 & $3021-3079 \mathrm{~s}$ & $3001-3002 \mathrm{~s}$ & $0.91-0.7$ \\
\hline Patient 18 & $298-320 \mathrm{~s}$ & $285-286 \mathrm{~s}$ & $0.9-0.86$ \\
\hline Patient 19 & $272-397 \mathrm{~s}$ & $242-243 \mathrm{~s}$ & $0.9-0.8$ \\
\hline Patient 20 & $2065-2175 \mathrm{~s}$ & $2040-2042 \mathrm{~s}$ & $0.9-0.75$ \\
\hline
\end{tabular}

\section{FUTURE DEVELOPMENTS}

Ongoing research aims at developing open-loop seizure system, intervention systems, implant devices, BCI alarm systems and Vagus Nerve Stimulators. [11]

These stimulation devices aid in correcting and treatment of the epilepsy, however do not facilitate the prevention of the occurrence of seizures. Thus, suitable algorithms that can provide control by predicting the onset of the seizure would give a novel solution for the arrest of epilepsy and improve the neurological health of the patient.

The electronic system designed will be a wearable device designed using the optimal prediction algorithm which predicts the occurrence of epilepsy seconds in advance. As soon as the device (brain pacemaker) detects the symptoms, it transmits electrical signals. The signals are sent to a wireless receiver to produce control signals for switching an alarm device, stimulator and take preventive actions. [10] 


\section{CONCLUSION}

Hence, it is evident that the quantitative approach using fractal analysis for identifying the onset of epileptic seizures in the epilepsy during the evaluation of the neurosurgery effects provides a promising computational tool for determination of the electrographic seizure onset in clinical applications. The application of a wavelet-based functional mixed-effect model for analysis of the cerebral and cerebellar activity in acute seizures can provide researchers with more details from the aspect of not only a prediction but also termination of epileptic attacks. The algorithm proposed, without errors can be implemented to develop closed-loop intervention brain pacemakers to automatically correct and prevent seizure attacks.

\section{ACKNOWLEDGEMENT}

We would like to thank Physio Net for permitting us to use their database.We also like to thank Dr. S. Shenbaga Devi, Professor, Department of ECE, College of Engineering Guindy,Anna University,Chennai for her valuable feeback and insightful comments.

\section{REFERENCES}

[1] C. Dilorio et al., "The epilelpsy medication and treatment complexity index: Reliability and validity testing," Journal of Neuroscience Nursing, pp. 155-158, June 2003.

[2] F. Mormann, R. G. Andrzejak, C. E. Elger, K. Lehnertz, "Seizure prediction: the long and winding road," Department of Epileptology, University of Bonn, Bonn, Germany.

[3] A. L. Goldberger, L. A. N. Amara, L. Glass et al., "Stanley HE. PhysioBank, PhysioToolkit, and PhysioNet: Components of a New Research Resource for Complex Physiologic Signals," Circulation vol. 101 , no. 23 , pp. 215 -e220

[4] A. Tanner, "Automatic seizure detection using a two-dimensional EEG feature space," thesis submitted for examination for the degree of Master of Science in Technology, 2011.

[5] M. D'Alessandro et. al., "Epileptic Seizure Prediction Using Hybrid Feature Selection Over Multiple Intracranial EEG Electrode Contacts: A Report of Four Patients," IEEE Transactions on Biomedical Engineering, vol. 60, issue 5, pp. 603-615, May 2003.

[6] J. Dauwels and E. Eskandar, "Localization of seizure onset area from intracranial non-seizure EEG by exploiting locally enhanced synchrony," in Proc. IEEE Eng Med Biol Soc., 2009, pp. 2180-2183.
[7] M. Isfan, R. Menezes, and D. A. Mendes, "Forecasting the Portuguese Stock Market Time Series by Using Artificial Neural Networks," Journal of Physics: Conference Series, vol. 221, pp. 12-17, 2010

[8] O. Morales-Matamoros, T. I. Contreras-Troya, C. I. Mota-Hernández, and B. Trueba-Ríos "Fractal Analysis of Epilepsy," in Proc. the 53rd Annual Meeting of the ISSS, Brisbane, Australia, 2009.

[9] K. P. Indiradevi, E. Elias, and P. Sathidevi, "Complexity analysis of electroencephalogram records of epileptic patients using Hurst exponent," International Journal of Medical Engineering and Informatics, vol. 1, no. 3, pp. 368, 2009.

[10] R. Brooks, "Toward a Brain-Internet Link," Wireless News Factor, 10 Dec 201.0

[11] D. Krieger and B. Litt, Seizure Prediction: Its Evolutionand Therapeutic Potential, Elsevier, 2001.

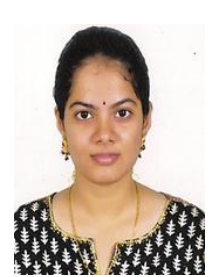

Kavya Devarajan has pursued B.E Biomedical Engineering in College Of Engineering. She has been actively involved in various biomedical projects in Digital Image Procesing, She has also presented a paper on "Brain Gate and its Applications" at an inter-college level. She has undergone training at Singapore Science Centre.

S. Bagyaraj has done B.E in Electronics and Instrumentation Engineering from Periyar University and M.E in Medical Electronics from Anna University. At present he is a Teaching Research Associate in the department of ECE, Centre for Medical Electronics at College of Engineering Guindy, Anna University. His research interests are in Cognitive Neuroscience, Functional analysis of brain using Near Infrared Spectroscopy, Rehabilitation Engineering. He is a life member in Biomedical Engineering Society of India.

E. Jyostna has done her undergraduate in Biomedical Engineering at College Of Engineering, Guindy,Anna University. She has completed projects on Segmentation of retinal blood vessels, respiration rate meter.

K. Jayasri is a budding Biomedical Engineer from College of Engineering ,Guindy, Anna University. As a part of her B.E program,she has done projects on "EEG Based Epilepsy Detection And Prediction."

Vinitha Balasampath is currently in her final year of B.E. Biomedical Engineering at the College of Engineering Guindy, Anna University,Chennai,India.She has also successfully worked individually on a mini project to develop a cost effective Phonocardiogram(PCG) and also a project on "Extraction of Region of Interest, Segmentation and Compression of An Image". 